Jean Célestin Ky

Jean Célestin Ky a étudié à l'Université de Paris I PanthéonSorbonne et est Maître-assistant en Histoire de l'art à l'Université de Ouagadougou, Burkina Faso. E-mail: Jean_ky@univ-ouaga.bf et kycelestin@yahoo.fr

\title{
A propos des arts plastiques à la
Semaine Nationale de la Culture \\ A propos des arts plastiques à la
Semaine Nationale de la Culture
}

\section{A propos des arts plastiques à la Semaine Nationale de la Culture}

\begin{abstract}
Le Burkina Faso organise tous les deux ans à Bobo Dioulasso, la Semaine Nationale de la Culture dont les objectifs essentiels demeurent la valorisation de la culture et la promotion des artistes burkinabè. Cette manifestation biennale, si elle concerne nombre de domaines des arts, reste un cadre de valorisation et de promotion très perceptible pour les arts du spectacle surtout. Les arts plastiques ne semblent pas y avoir trouvé une voie d'expression et de promotion pour leur plein épanouissement. Les facteurs militant contre cette catégorie d'art sont la confusion entre art traditionnel et art contemporain, I'anonymat de l'artiste plasticien, I'absence d'un thème autour duquel organiser la compétition, le sort non enviable réservé aux œuvres primées et aux artistes lauréats. Des propositions de solutions sont faites, espérant qu'elles produisent l'envol des arts plastiques à partir de la Semaine Nationale de la Culture. Mots clés: Semaine Nationale de la Culture burkinabè, Art plastique burkinabè, Plasticiens burkinabè, Promotion des arts plastiques burkinabè, Développement artistique au Burkina Faso.
\end{abstract}

Au Burkina Faso, la Semaine Nationale de la Culture (SNC) se déroule tous les deux ans à Bobo-Dioulasso, la seconde ville du pays. Il s'agit d'une manifestation culturelle dont le volet compétition met en présence d'innombrables artistes dans les domaines que sont les arts du spectacle, les arts culinaires, le sport traditionnel et les jeux de société, la littérature et les arts plastiques, etc.

Depuis sa création jusqu'à nos jours, la SNC a enregistré la participation des arts plastiques et des artistes plasticiens. Mais cette manifestation culturelle, telle qu'organisée et vécue par les artistes, accorde une importance et des privilèges notables à certaines expressions artistiques. Cet état de fait conduit à des interrogations au sujet des arts plastiques, mais qui pourraient être généralisées pour concerner d'autres domaines de l'art. Ainsi, passée une vingtaine d'années après la première édition de la SNC, on peut se poser les questions suivantes :

- la SNC a-t-elle favorisé les expressions plastiques?

- a-t-elle promu les artistes plasticiens?

- quelle place y occupent les arts plastiques et les artistes plasticiens? 
L'organisation de la SNC est un thème très récent de la vie culturelle du Burkina Faso. Ainsi, l'essentiel des matériaux pour le traiter repose sur : les textes fondateurs de la manifestation; les rapports sur les éditions de la SNC; les rapports généraux des séminaires sur la SNC; les rares travaux (mémoires) sur la manifestation culturelle; les observations personnelles sur le terrain et les témoignages de quelques artistes.

Les résultats de notre réflexion que nous présentons ici sont structurés en deux parties distinctes. La première, celle des aspects généraux, concerne la création de l'institution, les objectifs recherchés et l'organisation des manifestations. Il ne s'agit pas véritablement d'une analyse mais d'un rappel qui annonce la seconde partie. La deuxième partie isole les arts plastiques et décrit leur participation aux éditions de la SNC, depuis les phases éliminatoires jusqu'aux phases finales. Elle identifie les entraves et les contraintes à la participation plus importante des artistes plasticiens. Par la même occasion, pour dynamiser les arts plastiques à la SNC, des solutions sont proposées.

\section{La Semaine Nationale de la Culture : aspects généraux}

Au Burkina Faso, les années 1980 voient naître des volontés de réformes en faveur de la culture. Au nombre d'elles, la SNC, créée par le Conseil du Salut du Peuple (CSP) en juillet 1983 et mise en exécution par le Conseil National de la Révolution (CNR) en décembre 1983. ${ }^{1}$ Les premiers textes créant la Semaine Nationale de la Culture sont l'Arrêté $\mathrm{N}^{\circ} 329 / \mathrm{ENAC/DGAC}$ et la Décision No 325/ENAC/DGAC du 29 juillet $1983 \mathrm{du}$ Ministère de l'Education Nationale, des Arts et de la Culture (Traoré-Sanogo 1996 : 24). Il s'agit d'une initiative du Conseil du Salut du Peuple (CSP) qui se propose d'organiser le Grand Prix National des Arts et des Lettres et d'autres manifestations à caractère culturel. Mais renversé quelques jours plus tard, le CSP n'aura aucune chance d'organiser cette manifestation culturelle. Le Conseil National de la Révolution (CNR) lui succède et organise en décembre 1983 la première édition de la SNC à Ouagadougou du 20 au 30 décembre. La Révolution élucide le texte portant création et organisation de la SNC en fonction de sa vision de la culture exprimée dans le Discours d'Orientation Politique (DOP) du 2 octobre 1983, c'est-à-dire le discours - programme du Conseil National de la Révolution prononcé le 2 octobre 1983 à la Radio Télévision Nationale par le Président Thomas Sankara. La SNC est envisagée comme un engagement pour l'affirmation de l'identité culturelle burkinabè. Le Décret Nº4250/CNR/PRES du 27 juin1984 décide de l'organisation annuelle de ladite manifestation. C'est ce qui justifie le déroulement à Gaoua en 1984 de sa seconde édition. Mais, à l'issue des manifestations, les problèmes d'infrastructures culturelles et d'infrastructures d'accueil et d'hébergement conduisent les autorités à revoir la périodicité de la SNC; intervient donc le troisième texte, le Décret $\mathrm{N}^{\circ}$ 85-486/CNR/PRES/MIC du 29 août 1985 qui fait de la SNC une manifestation biennale. Cela pour permettre aux 
provinces hôtes d'avoir deux ans de préparation, le temps de se doter d'infrastructures culturelles et de logements. C'est dans cette vision que se tiennent les SNC Bobo 86 et Koudougou-Réo 88.

L'année 1990 marque un tournant très important dans la vie de la SNC. Le 15 octobre 1987, un coup d'Etat militaire, au terme duquel le Président Sankara et ses partisans trouvent la mort, instaure le Front Populaire (FP) et engage le pays dans un mouvement dit de Rectification. Le Capitaine Blaise Compaoré qui accède au pouvoir à cet effet conduit un régime d'exception jusqu'en 1991. Pendant cette période d'importantes décisions relatives à la vie de la biennale culturelle sont prises : la SNC se sédentarise à Bobo-Dioulasso, désormais seule localité à abriter les manifestations; elle est administrée par un Secrétariat Permanent basée dans cette ville. L'Arrêté Nº137/MIC/MDC/SP-SNC du 25 juillet 1991 fixe les attributions, l' organisation et le fonctionnement de la structure chargée de la SNC et chaque édition de la SNC est régie par un Arrêté ministériel qui lui donne sa légitimité. ${ }^{2}$ Ces mesures enracinent la SNC et en font la plus grande manifestation culturelle nationale jamais égalée au Burkina Faso. Cependant, de 1990 à nos jours, des séminaires de réflexion sur la SNC sont tenus en vue d'en identifier les acquis, pour les conserver ou les consolider, et les insuffisances, pour les corriger. ${ }^{3}$

\section{Objectifs et organisation}

Les recherches attestent que le Burkina Faso comprend une soixantaine d'ethnies ayant souvent chacune des expressions culturelles propres. Pour mieux dégager et comprendre les objectifs assignés à la SNC, il convient de faire un rappel historique. L'état actuel des connaissances permet de définir trois grandes phases de l'histoire du peuplement du Burkina (Kouanda 1986: 50) : le peuplement antérieur au XVe siècle qui est celui des Bobo, Bwa, San, Yônyôosé, Nînsi, Gurunsi, Dogon, Kurumba et Bisanô (Sing. Bisa); le peuplement entre le XVe et le XVIIe siècle qui est celui des Moose, Gulmanceba, Fulbé, Yarsé, Marka, Zara; et le peuplement à partir de la fin du XVIIe siècle qui est celui des Lobi, Dagara, Hausa, Dyula. A partir de ces trois vagues de peuplement, les populations ont été réparties en deux groupes : les autochtones ou populations anciennement installées (groupe qui comprend les populations dont l'installation s'est faite avant le XVe siècle) et les populations venues d'ailleurs (groupe qui se compose des populations installées à partir, XVe siècle).

La conquête des territoires du Burkina commencée en 1896 s'achève avant 1900. Mais le Burkina ne sera érigé en colonie qu'en 1919. Cela dure jusqu'en 1932, où pour diverses raisons, on assiste à sa dissolution. En 1947 intervient la reconstitution de la colonie de Haute-Volta dans ses limites de 1932. Elle entre dans la communauté française en votant oui au référendum du 28 septembre 1958. Le 11 décembre 1958 elle devient une République avant d'être indépendante le 5 août 1960. De 1900 à 1960, ce qui 
correspond à la période coloniale où les territoires du Burkina sont sous tutelle française, on assiste à une campagne de négation des pratiques culturelles locales au profit de celles du colonisateur. Ainsi, au dynamisme culturel et au sentiment de fierté culturelle remarquable précoloniaux succède un questionnement des valeurs culturelles. La civilisation française par l'école et le christianisme déstabilise divers domaines de la culture burkinabè. C'est dans ce contexte de négation du socle culturel burkinabè qu'intervient la proclamation de l'indépendance le 5 août 1960.

Quelles sont les dispositions prises par l'Etat en vue de la réhabilitation de la culture burkinabè? De 1960 à 1983, si la promotion de la culture a été une préoccupation des différents pouvoirs publics qui se sont succédés, on ne note pas chez eux une volonté politique de faire de la culture une dimension essentielle de l'homme, le socle du développement. L'inexistence jusqu'à une période récente d'un ministère autonome de la culture conforte quelque peu cette vue. Les politiques culturelles se limitent à quelques actions parmi lesquelles on peut relever la création du Musée National, la naissance d'un cinéma et l'érection d'une direction chargée du patrimoine culturel. Le Musée National voit le jour par la loi N42/62/AN du 13 novembre 1962 (Traoré 1985/1986 : 18) avec la mission d'être un espace de sauvegarde et de conservation de la mémoire collective du peuple burkinabè. C'est encore en 1962 que se crée une cellule cinéma rattachée à la Direction Générale de l'Information (TraoréSanogo 1996 : 21). Sa mission a consisté en la réalisation de films socio-éducatifs didactiques et d'actualités à l'intention de la population rurale notamment. ${ }^{4} \mathrm{Il}$ a fallu attendre 1972, dix ans après le Musée national, pour que se crée la Direction des Arts, des Lettres et du Patrimoine Culturel (Yaméogo 1989) avec une tutelle administrative assurée par le Ministère de l'Education Nationale.

En réalité, le tutorat qui a caractérisé pendant longtemps la gestion de la culture au Burkina n'a pas favorisé très tôt l'éclosion d'une véritable politique culturelle ou la naissance d'une vision en la matière. D'abord inexistante dans les organigrammes des départements ministériels (de 1960 à 1971), la culture tantôt rattachée au ministère de l'Education Nationale, tantôt rattachée au Ministère de la Jeunesse et des Sports sera plus animée par des initiatives privées que par une orientation politique gouvernementale déterminée. C'est pourquoi, si en 1983, au niveau du cinéma, des efforts notables sont réalisés, il n'en n'est pas de même pour les autres domaines de la culture. En effet, les arts du spectacle, les arts plastiques, la littérature et l'artisanat, pourtant des domaines essentiels de la culture burkinabè, sont insuffisamment promus ou ne le sont même pas. C'est dans ce contexte qu'intervient un changement de régime en 1983.

Le pouvoir issu du coup d'Etat militaire du 4 août 1983 a reconnu l'importance de la culture dans le processus de transformation du Burkina Faso. ${ }^{5}$ Cette culture qui a un caractère national devrait porter toutes les expressions culturelles et tous les aspects du patrimoine culturel. Cette revalorisation de la culture vise la formation d'une 
identité culturelle indispensable à la prise de conscience de sa propre valeur et à la confiance en soi. On peut, sans grand risque de se tromper, affirmer que de 1983 à nos jours on voit s'affirmer de plus en plus une volonté politique de réhabilitation de la culture burkinabè et $\mathrm{d}$ 'installation du peuple burkinabè dans sa culture. Nous n'analyserons pas ce processus ici, ce n'est pas le but avoué de ce point, nous avons surtout en vue l'identification des objectifs qui fondent la SNC.

Nous l'avons vu, la SNC est une initiative du CSP que le CNR rend manifeste, en organisant quatre mois après son accession au pouvoir, du 20 au 30 décembre 1983 la première édition de la Semaine Nationale de la Culture. Et depuis ce temps, cette manifestation, victorieuse des différentes épreuves qui se sont présentées à elle, se déroule toujours. En effet, en parcourant les textes fondateurs de la SNC et les rapports des divers séminaires organisés sur cette institution, on note une constance dans les objectifs qu' on lui assigne. En faisant une synthèse, ils sont les suivants : la dynamisation de la vie culturelle nationale; la revalorisation et la redécouverte du patrimoine culturel et artistique; l' affirmation de l'identité culturelle; le brassage des différentes identités culturelles et la promotion des artistes. En clair, selon les textes, la SNC est le cadre qui favorise l'expression de tous les domaines des arts de la culture burkinabè et fait la promotion des artistes les meilleurs.

Comment cette manifestation biennale s'organise-t-elle? Dans le processus d'organisation de la SNC, les éliminatoires qui constituent la phase préliminaire se déroulent en différentes étapes. D'abord la possibilité est offerte à chaque département d'organiser les journées départementales de la culture. ${ }^{6}$ La compétition à ce niveau concerne les villages qui forment le département et permet de retenir les meilleurs ensembles artistiques et les meilleures œuvres d'art. Ensuite viennent les journées provinciales de la culture qui mettent en compétition les troupes et les objets des divers départements de la province concernée. Al'issue des journées provinciales, chaque province retient ses meilleurs troupes et objets d'art pour la compétition au niveau régional. La Semaine Régionale de la Culture fait l'objet d'un Arrêté ministériel et est organisée par une province hôte avec l'appui technique et financier du Secrétariat Permanent de la SNC. A cette phase, des jurys nationaux, constitués sur proposition du Secrétariat, retiennent par région les meilleurs ensembles artistiques, œuvres d'art et plats cuisinés (art culinaire) présentés par les provinces.

Ces différentes sélections débouchent sur la phase finale : la grande manifestation culturelle qui a lieu à Bobo-Dioulasso pendant une semaine. L'étape finale, vitrine de la culture burkinabè, comprend un volet compétition et un volet festival. Dans le cadre de la compétition, les artistes du spectacle, les artistes plasticiens, les écrivains, les cinéastes et bien d'autres, livrent une ultime compétition appelée le Grand Prix National des Arts et des Lettres (GPNAL); les compétitions en art culinaire et en sports traditionnels en sont exclus. ${ }^{7}$ Ces compétitions finales permettent la désigna- 
tion, à la clôture, des meilleurs artistes et écrivains du Burkina Faso. Quant au volet festival, il permet la découverte de différents aspects de la culture ne faisant pas l'objet d'une compétition. Ces manifestations comprennent les défilés et danses des masques, les exhibitions hippiques, la présentation d'art vestimentaire, la foire, ${ }^{8}$ les expositions muséales et d'art plastique, ${ }^{9}$ les activités récréatives et de loisirs dont le village des communautés,$^{10}$ les espaces pour enfants (activités de formation, jeux concours), les animations et les bals populaires, les activités touristiques, les colloques, séminaires et conférences.

Toutes ces activités et manifestations participent à l'animation de la SNC et en font une véritable fête vécue par les festivaliers et d'autres populations par la retransmission en direct par la Télévision nationale des compétitions du GPNAL en arts du spectacle et par des reportages sur certaines animations. Voici venu le moment de voir la place des arts plastiques dans le volet compétition de la SNC.

\section{Les arts plastiques (1983-2004)}

Le but de cette partie est de relever et révéler la participation des arts plastiques aux éditions de la SNC en vue d'en identifier les insuffisances et les obstacles à une véritable promotion de ce domaine de l'art. La participation des arts plastiques à cette manifestation comprend les expositions des artistes à la foire à des fins commerciales, les expositions muséales et la compétition au GPNAL. C'est cette dernière, la compétition en arts plastiques, qui est en débat ici et qui fait l'objet de la présente réflexion.

\section{La compétition en arts plastiques}

Dans les compétitions des GPNAL de la SNC les arts plastiques font partie de la catégorie B. ${ }^{11}$ Les arts plastiques sont ceux qui sont producteurs ou reproducteurs de formes ou de volumes; c'est l'art de la représentation des formes. La classification traditionnelle des arts retient comme arts plastiques l'architecture, la sculpture et la peinture. Cependant, au Burkina Faso, la notion d'art plastique est plus large. La réalité a conduit à l'ajout de certaines pratiques, notamment des techniques qui donnent des objets d'art. Ainsi, dès 1983 ont été retenues comme disciplines des arts plastiques la sculpture, la peinture, le bronze, le batik, la photographie, la maroquinerie, le tissage, l'art vestimentaire, l'art capillaire, le dessin, le modelage et la vannerie.

Ces différents domaines resteront inscrits au titre des compétitions dans les arts plastiques jusqu'à la SNC 1996. En effet, l'Arrêté ministériel portant Règlement Intérieur du GPNAL de la catégorie B à la SNC 1998 retient au titre des arts plastiques la sculpture, la peinture, le batik, le bronze et le dessin pour les enfants qui, plus tard, disparait ${ }^{12}$ (Cf. l'Arrêté n ${ }^{\circ}$ 99-0011/MCC/SG/SP-SNC, I'Arrêté n²003-395/MCAT/SG/SPSNC et l'Arrêté n²005-201/MCAT/SG/SP-SNC). Dès la publication du règlement in- 
térieur, les artistes s'inscrivent dans les disciplines relevant de leurs spécialités en vue des phases éliminatoires. Il semble que, concernant les arts plastiques, les éliminatoires au niveau des départements et des provinces ne se déroulent pas souvent. Cette impression repose sur un témoignage et un constat. En effet, en octobre1997, lors des sélections régionales qui se sont déroulées à Dédougou, un assistant culturel de la province du Nayala nous confie que les pièces de cette province n' ont pas fait l'objet d'une sélection avant leur présentation dans la région. Aussi, étant membre du jury des sélections régionales, nous voyons certains artistes se présenter eux-mêmes avec les objets en lieu et place des responsables culturels de la province. C'est dire que le premier niveau de compétition en arts plastiques commence véritablement au niveau de la région culturelle.

Les premières sélections régionales se déroulent à partir de la deuxième édition de la SNC (Traoré-Sanogo 1996 : 52). Jusqu' aux sélections régionales de la SNC 2004 qui se déroulent en 2003, le Burkina Faso se divise en cinq régions culturelles comprenant chacune un ensemble de provinces, auxquelles s'ajoutent les régions autonomes $\mathrm{du}$ Houet et du Kadiogo ainsi que la diaspora en Côte d'Ivoire. Mais, à partir de cette année, le Burkina Faso est divisé en quatorze régions culturelles. En effet, les sélections régionales en arts plastiques ont connu une modification qui ne semble pas favoriser sa pleine expression. Si jusqu'en 1999 chaque région recevait le jury de sélection, à partir de 2001 les régions en arts plastiques se limitent aux provinces autonomes du Houet et du Kadiogo. C'est dire que les sélections des œuvres dans la catégorie B s'effectuent en deux zones, l'une dans la province du Houet et l'autre dans la province du Kadiogo, dans lesquelles les œuvres parviennent selon une répartition. Là, les trois premières œuvres ayant obtenu au moins 13/20 de moyenne dans chacune des disciplines sont retenues pour la phase finale. ${ }^{13}$ En faisant un calcul on passe d'un maximum de quatre-vingt-quatre (84) objets à un maximum de vingtquatre (24) en compétition à la phase finale.

Les œuvres d'art sont reçues par le Secrétariat Permanent de la SNC et remises à la commission exposition. Cette commission les expose dans une salle en attribuant à chaque objet un code d'anonymat. Selon les témoignages, au moment où les arts plastiques intégraient la sculpture, la peinture, le bronze, le batik, la photographie, la maroquinerie, le tissage, l'art vestimentaire, l'art capillaire, le dessin, le modelage et la vannerie avec sept sélections régionales, arrivait à la phase finale une centaine d'œuvres à examiner. Mais, avec la réduction des disciplines des arts plastiques limitées à la sculpture, la peinture, le bronze et le batik, n'arrive à ce stade de la compétition qu'une cinquantaine d'objets (Cf. rapport du jury de la catégorie B du GPNAL de la Xe édition de la SNC). Et la limitation des régions culturelles à deux (2) pour cette catégorie engendre à nouveau une réduction du nombre d'objets à la phase finale; la dernière édition n'enregistre que vingt-cinq (25) objets (Cf. procès-verbal de délibération du jury de la catégorie B SNC Bobo 2004). 
C'est à la phase finale que sont sélectionnés et primés les meilleurs objets. Ce travail revient à un jury nommé par Arrêté du Ministre chargé de la culture sur proposition du Secrétariat Permanent de la SNC. Le jury national se compose de cinq (5) membres qui sont autonomes et souverains. Avant de commencer leur travail, les membres du jury définissent et harmonisent les critères d'appréciation et les points à leur attribuer. ${ }^{14}$ Régulièrement les éléments suivants reviennent : $l^{\prime}$ originalité, la créativité, l'esthétique, la composition, l'exécution, la finition et la pertinence du thème. $C^{\prime}$ est à partir des critères retenus que les œuvres toujours sous anonymat sont notées par chaque membre du jury. Et c'est la moyenne des notes attribuées à chaque objet qui détermine son rang général. Le jury ne lève l'anonymat que lorsque vient le moment du classement en fonction des moyennes obtenues. Ne sont citées au palmarès officiel et primées que les trois premières œuvres par discipline ayant obtenu une moyenne au moins égale à 13/20. Elles font de ce fait partie du patrimoine national burkinabè à travers leur acquisition par l'Etat. Quelle analyse faire de la compétition en arts plastiques à la SNC?

\section{Analyse de la participation des arts plastiques à la Semaine nationale de la culture}

Rappelons que, pour l'essentiel, la SNC vise la mise en valeur de tous les aspects de la culture burkinabè et la promotion des artistes. Dans le cas des arts plastiques, force est de constater aujourd'hui que la SNC n'a ni développé tous les domaines y afférents ni fait la promotion des artistes plasticiens; la baisse progressive du nombre de participants conforte cette thèse. Lorsqu'on examine, même sans grande minutie, la compétition dans catégorie à la SNC, on relève des insuffisances qui, si elles ne justifient pas, expliquent le peu d'intérêt ou le recul de cette discipline.

La SNC ne fait pas de distinction entre art traditionnel et art contemporain dans la catégorie $\mathrm{B}$, contrairement aux arts du spectacle où existent la danse traditionnelle, la musique traditionnelle et la vedette de la chanson traditionnelle. En effet, le domaine de l'art contemporain utilise des matériaux, des outils et des techniques plus modernes; à côté de cela, l'art traditionnel avec tous les éléments qui le caractérisent. Il ne nous semble pas pertinent que les mêmes critères d'appréciation soient appliqués à ces deux types d'art très différents. Cette absence d'un volet art plastique traditionnel défavorise les artistes plasticiens travaillant selon les canons culturels traditionnels. Les critères de jugement étant en faveur des arts contemporains, les lauréats ont toujours été les artistes travaillant en milieu urbain dans des ateliers et issus des centres de formation. Avec le temps, les artistes ruraux finissent par comprendre que la compétition de la catégorie B n'est pas faite pour eux.

Cette sorte d'exclusion des artistes traditionnels est un obstacle à la valorisation des arts plastiques en rapport avec la culture burkinabè. En effet, au Burkina Faso comme ailleurs en Afrique, les œuvres créées portent l'histoire des groupes qui, pour matérialiser les formes, gardent leurs normes culturelles. C'est pourquoi ils ont dif- 
férentes possibilités plastiques de représenter les mêmes images. Ainsi Moose, Nuna, Léla, Bwa, Bobo, Winiéma, Marka, Kurumba et autres possèdent chacun ses propres conventions pour représenter l'antilope ou le buffle par exemple. Pour conserver et valoriser tous les atouts plastiques traditionnels, il convient d'organiser les compétitions en arts plastiques autrement. La catégorie B comprendra deux volets que sont l'art traditionnel et l'art contemporain. L'art traditionnel renferme la sculpture (bois, bronze), la maroquinerie, le tissage, l'art vestimentaire, l'art capillaire, la poterie, la vannerie et la peinture (Pibot 2001). L'art contemporain englobe la sculpture (bois, bronze, pierre, fer), le batik, le design et les arts composites et la peinture (Kaboré 1992/ 1993; Eliard 2002).

A l'absence des canons culturels traditionnels s'ajoute l'anonymat de l'artiste plasticien; à toutes les étapes de la SNC aucune occasion n'est offerte à l'artiste plasticien de se faire connaître. Les visiteurs qui, dans la salle d'exposition, apprécient favorablement une œuvre et souhaitent entrer en contact avec l'auteur, ne peuvent le faire du fait de l'anonymat. L'anonymat qui caractérise sa participation ne lui laisse aucune chance de rencontre avec des artistes étrangers organisateurs de symposiums ou d'expositions-ventes internationales. Ainsi, l'artiste au soir de la SNC retourne chez lui sans le moindre contact pouvant lui ouvrir de nouveaux horizons. Pourtant, les artistes du spectacle profitent de la SNC pour rencontrer les promoteurs de spectacles étrangers surtout, ce qui aboutit souvent à des déplacements à l'extérieur (TraoréSanogo 1996: 74-77). Pour lever l'anonymat et mieux présenter l'artiste plasticien au public, la phase finale de la SNC est à organiser autrement. Il faut envisager la création d'un site des arts plastiques où travailleront sur place tous les artistes en compétition. On aura ainsi une place vivante où pourront être appréciés les artistes et les œuvres d'abord en production puis produites.

Mais l'absence de thème précis à partir duquel travaillent les artistes complique le travail de sélection. En effet, les œuvres d'art de la SNC, même celles d'une même discipline, abordent des thèmes différents rendant malaisée toute attribution de notes. Face à des tableaux de peinture qui abordent des thèmes différents, l'appréciateur pourrait, au-delà de la qualité plastique de l'œuvre, être attiré par le thème qui l'intéresse. Ce risque ne place pas les œuvres à un même niveau d'appréciation rendant inégale leur chance d'être citées au palmarès officiel. Pour l'éviter, il convient de retenir un thème unique par édition à partir duquel les artistes fabriqueront leurs œuvres. ${ }^{15}$ Ce thème sera le premier critère de jugement des œuvres en compétition.

La question du devenir des œuvres primées est aussi importante. Les textes, malgré leur clarté sur cet aspect, ne sont pas appliqués. Il semble qu'au début, nous ne l'avons pas vérifié, toutes les œuvres primées deviennent la propriété de l'Etat par leur acquisition par le Musée National. Ainsi, les premiers lauréats voient leurs productions conservées au Musée National. Par la suite, cette disposition évolue pour dire que les œuvres primées sont achetées par l'Etat au profit du Musée National ou 
encore les œuvres classées premières dans chaque discipline sont d'office achetées par le ministère en charge de la culture au profit du patrimoine culturel du Musée National (Cf. Arrêté Nº9-0011/MCC/SG/SP-SNC, portant Règlement intérieur du Grand Prix National des Arts et des Lettres, 10ème édition de la Semaine Nationale de la Culture SNC BOBO 2000). ${ }^{16}$ Les œuvres n'étant pas souvent acquises par $l^{\prime} E t a t$, abandonnées aux mains de leurs propriétaires, retournent dans leurs provinces d'origine ou sont vendues sur place à d' autres acquéreurs.

Celles qui parviennent au Musée national ne connaissent pas une situation enviable. Mal conservés et non mis en valeur, ces objets ne peuvent faire connaître leurs créateurs. En effet, une récente recherche a établi l'inexistence de salles de réserves, de méthode, moyens et techniques de conservation au Musée national mettant ainsi en danger la vie des objets (Bayala 2004/2005). Pourtant, les représentations primées en arts du spectacle et les œuvres primées en littérature connaissent une situation bien meilleure. Sous réserve de contrôle, des cassettes sont produites pour les meilleurs ensembles artistiques musicaux; ce sont eux aussi qui, périodiquement, participent à la «Caravane du Sahel » qu'organise le Ministère de la Culture, des Arts et du Tourisme. ${ }^{17}$ Quant à la littérature, le GPNAL qui récompense à chaque édition les meilleures œuvres littéraires, reste une occasion de se faire éditer. Le tableau des ouvrages édités dans ce cadre par le Ministère en charge de la culture est déjà important (Cf. TraoréSanogo 1996 : 65).

Pour ce qui est des productions plastiques, leur devenir s'exprime en terme de conservation et de mise en valeur. Ces objets ont besoin d'une meilleure conservation qui les protège de toute détérioration et d'une mise en valeur. Le meilleur cadre de protection et conservation d'un objet ayant un intérêt artistique, culturel ou historique est le musée. Le Secrétariat Permanent de la SNC n'en ayant pas, le Musée National, sous réserve d'amélioration de leurs conditions de séjour, peut continuer à recevoir les meilleurs objets du GPNAL des éditions de la SNC. Leur mise en valeur devra se traduire par des expositions permanentes ou temporaires (au Musée National ou à la SNC), itinérantes à l'occasion surtout des sélections régionales et à l'extérieur lors des tournées de la «Caravane du Sahel » afin de les révéler au public et rappeler en même temps leurs auteurs. En réalité, pour leur mise en valeur, plusieurs usages peuvent être faits des meilleures œuvres d'art de la SNC dont la décoration et l'ornement des bâtiments administratifs, l'impression comme motifs sur les pagnes et les tricots notamment ceux de la SNC, l'impression des timbres postaux, etc.

Notons enfin l'absence de promotion des artistes plasticiens primés. Il nous semble qu'en dehors des prix servis, les artistes plasticiens ne reçoivent aucune faveur qui puisse les promouvoir. Sous la révolution, entre 1983 et 1987, $1^{\prime}$ Etat traduit une volonté de réformes en faveur des artistes plasticiens lauréats; ils bénéficient de commandes très importantes venant du pouvoir. Ainsi, l'artiste-peintre Raya Benjamin Sawadogo, lauréat de la première édition de la $\mathrm{SNC}$, obtient la décoration du marché 
central de Ouagadougou et le sculpteur bronzier Ali Nikiéma lauréat des éditions de 1983, 1984 et 1988 reçoit des commandes pour les armoiries du Faso en 1984, le monument de la bataille du rail à Ouagadougou en 1986 et celui de la jeunesse à Ziniaré en 1987 (Traoré-Sanogo 1996 : 70-73). Cette disposition de promotion des artistes lauréats, aussi intéressante soit-elle, ne se poursuit pas. Cette reconnaissance d'une qualité ou d'un travail bien fait ne serait pas inintéressante à rétablir afin que les grands marchés publics et privés en arts plastiques s'exécutent par les lauréats du moment. En outre, ces derniers peuvent intervenir en qualité de formateurs dans les centres de formation en arts plastiques, par exemples le Centre National d'Artisanat d'Art et le Village Artisanal de Ouagadougou.

\section{Conclusion}

Ce travail est une réflexion personnelle de nos différentes participations aux jurys des sélections régionales et des phases finales de la SNC. Nos observations ont abouti au constat ferme qu'à cette manifestation biennale tous les arts n'ont pas la même importance. En effet, créée pour être un cadre de valorisation et de promotion de la culture burkinabè, la SNC semble accorder la prééminence et la primauté à des aspects précis de la culture. Ainsi, de manière très perceptible, les arts du spectacle constituent l'essence de la SNC. La place qu'ils occupent dans les trois temps de la SNC (avant, pendant et après) conforte cette vision.

Au nombre des arts qui n'y trouvent pas encore une voie d'expression et de promotion en vue de leur plein épanouissement, il y a les arts plastiques. On pourra alors constater la faible participation ou le peu d'intérêt des artistes plasticiens pour la SNC dont il faut rechercher les causes ou qu'il faut analyser. Les facteurs militant contre cette catégorie sont la confusion entre art traditionnel et art contemporain, l'anonymat de l'artiste plasticien, l'absence d'un thème autour duquel s'organise la compétition, le sort non enviable réservé aux œuvres primées et aux artistes lauréats.

La limitation de la culture aux arts du spectacle ou le privilège qui leur est accordé ne favorise ni ne valorise toutes les expressions culturelles comme le veut le thème de la SNC BOBO 1998 : "Favoriser et valoriser la rencontre des expressions artistiques". Comme les arts plastiques, le traitement de l'art culinaire, de la littérature et de bien d'autres mérite d'être analysé; analyse desquelles partiront des propositions de solutions espérant qu' elles produisent l'envol de tous les arts ou de toute la culture burkinabè à partir de la SNC.

Notes

1. Le 7 novembre 1982 marque la fin du Comité Militaire de Redressement pour le Progrès National (CMRPN) dirigé par le Colonel Saye Zerbo depuis le 25 novembre 1980. En effet, les crises internes au CMRPN auxquelles se sont ajoutées des crises sociales aboutissent à la prise du pouvoir par le 
Conseil Provisoire du Salut du Peuple (CPSP), devenu plus tard Conseil du Salut du Peuple (CSP). Le CSP porte à la tête de l'Etat le Médecin Commandant Jean-Baptiste Ouédraogo et ce, jusqu'au 4 août 1983. Le 4 août 1983, le Conseil National de la Révolution (CNR) accède au pouvoir avec à sa tête le Capitaine Thomas Sankara, chef de file d'un groupe de jeunes officiers. D'une orientation révolutionnaire s'inspirant du marxisme-léninisme le CNR est renversé le 15 octobre 1987 suite à des querelles intestines entre le Président Sankara et le Capitaine Blaise Compaoré, la seconde personnalité du régime.

2. Jusqu'en 1990, la Semaine Nationale de la Culture n'était qu'une activité organisée par des directions du Ministère en charge de la culture; il s'agit d'abord de la Direction Générale des Affaires Culturelles (DGAC), puis de la Direction de la Promotion Culturelle (DPC) et enfin de la Direction des Arts du Spectacle et des Lettres (DASL).

3. C'est ainsi qu'en 1985, le Séminaire de Matourkou se déroule du 22 au 25 avril. Il contribue à jeter des bases d'une politique culturelle qui devrait jouer un rôle dans l'édification d'une conscience nationale. Le séminaire propose la revalorisation du patrimoine culturel, la promotion des artistes et des hommes de culture, la création d'un ministère de la culture, etc. En 1993, du 5 au 10 avril a eu lieu une rencontre avec pour thème : «La SNC : Quelle stratégie pour l'avenir? ». Après dix années d'existence, il fallait jeter un regard rétrospectif afin de cerner les acquis et envisager une nouvelle orientation (Cf. Rapport général du séminaire sur la SNC : 1993). Il en est de même pour la rencontre des experts à Bobo-Dioulasso en 1997 et le séminaire sur le redimensionnement de la Semaine Nationale de la Culture en octobre 2004.

4. Cela a été le point de départ de toute une politique de développement cinématographique au Burkina Faso. Aujourd'hui ce pays est arrivé à s'imposer comme centre du cinéma africain. On peut, sans entrer dans les détails, reconnaître que le Burkina a réussi sa politique de création d'un cinéma burkinabè et d'organisation du cinéma africain.

5. Dès sa prise du pouvoir le 4 août 1984, le Conseil National de la Révolution a tracé l'orientation générale de sa politique culturelle en affirmant son soutien à la culture et en créant les cadres permettant aux artistes et aux hommes de culture de se mouvoir et de libérer leurs génies. Le 2 octobre 1983, le Discours d'Orientation Politique (DOP) prononcé par le Président du Conseil National de la Révolution traduit cela en ces termes; «Quant à la culture, elle devra revêtir un triple caractère : national, révolutionnaire et populaire. La Révolution Démocratique et Populaire créera les conditions propices à l'éclosion d'une culture nouvelle. Nos artistes auront les coudées franches pour aller hardiment de l'avant. Ils devront saisir l'occasion qui se présente à eux pour hausser notre culture au niveau mondial ».

6. Le découpage du Burkina Faso en régions culturelles a évolué; de sept (7) régions au départ, nous en comptons aujourd'hui quatorze (14) dans lesquelles se dérouleront cette année les présélections régionales prévues du 17 novembre 2005 au 7 janvier 2006.

7. En 2004, l'art culinaire faisait partie intégrante du GPNAL, mais pour 2006 il ne l'est pas.

8. C'est en 1986 que l'on note véritablement le début des foires. Et depuis, la foire s'est imposée comme une activité incontournable visant la promotion des produits burkinabè. Les Burkinabè, à travers la foire, valorisent leurs potentialités ou leur savoir-faire dans les domaines des arts plastiques et culinaires, de l'artisanat, de la pharmacopée et voire de l'industrie. La foire est aussi le moyen d'atteindre des objectifs commerciaux à court et à moyen terme; les exposants peuvent exploiter toutes les opportunités commerciales pour la conquête du marché intérieur et sousrégional.

9. L'exposition muséale participe à l'expression et à la valorisation d'une culture. Dans le cas de la SNC, elle manifeste un pan de la réalité culturelle de notre pays. L'exposition de Bobo 98 portait sur le thème «l'image de la femme dans la statuaire burkinabè ». Elle a permis de mettre en exergue la place de la femme dans les diverses sociétés à partir d'une gamme variée de figurines en bois et en bronze. A la biennale 2000 une exposition sur le thème « instruments traditionnels de musique, fonction et évolution » a fait connaître une série d'objets artistiques traditionnels, élément de notre patrimoine culturel. Ces expositions sensibilisent les Burkinabè à mieux apprécier leurs richesses, à les valoriser.

10. Le village des communautés présente le vécu quotidien de celles-ci tel qu'il se déroule au village. Il présente un art de vivre des communautés rurales autour de la bière de mil ou du bandji (boisson alcoolisée extraite du rônier), de la musique, etc. Les animations de réjouissances populaires sous l'égide de la parenté à plaisanterie sont un puissant facteur de cohésion entre les diverses communautés culturelles. Le village des communautés magnifie la solidarité et entraîne le brassage culturel. 
11. Les GPNAL de la SNC ont concerné trois (3) catégories que sont les catégorie A pour les arts du spectacle B pour les arts plastiques, C pour les lettres; la reforme de 1997 introduit les catégorie D (Discours en langues nationales), E ou Pool jeune (créations artistiques par les jeunes) et $\mathrm{F}$ (cinéma et productions audiovisuelles).

12. Pour la 13ème édition de la SNC Bobo 2006, l'Arrêt ministériel indique que la catégorie B comporte les disciplines suivantes : la sculpture, la peinture, le batik et les arts composites.

13. Les nouveaux textes autorisent à retenir à ce niveau de la compétition les six (6) premières œuvres de chaque discipline.

14. Le règlement intérieur ne comportant aucun critère d'appréciation des oeuvres, il revient au jury de les déterminer.

15. Cela se fait pour les épreuves de dessin; les concours de logos ou les maquettes qui se font à partir des indications du commanditaire.

16. Le règlement intérieur de la SNC BOBO 2006 dit que les oeuvres primées au palmarès officiel sont propriété de l'Etat burkinabè. Une indemnité forfaitaire d'un montant de 150000 F CFA sera versée à chaque lauréat au titre des frais d'acquisition de son œuvre (Cf. Arrêté №2005-201/MCAT/SG/SP$\mathrm{SNC})$. [1 Euro $=655,957 \mathrm{~F} \mathrm{CFA}]$

17. La «Caravane du Sahel » c'est le déplacement à l'extérieur, dans les pays voisins surtout, des troupes de danse et de musique burkinabè en vue de faire voir et découvrir un aspect de la culture burkinabè. La « Caravane du Sahel » s'est déjà rendue en Côte d'Ivoire, au Mali, au Ghana, etc.

\section{Bibliographie}

Balima, S.-A. 1996. Légendes et histoire des peuples du Burkina Faso. Paris : Imprimerie de l'indépendant.

Bayala, E. 2004/2005. La gestion des collections du Musée National et la question de sécurité dans les réserves. Mémoire de maîtrise en Arts, Gestion et Administration Culturelle, Université de Ouagadougou, UFR/LAC, Filière AGAC.

Coquery-Vidrovitch, C. (sous la direction de). 1992. L'Afrique occidentale au temps des Français. Colonisateurs et colonisés, c. 1860-1960. Paris : La Découverte.

Eliard, S. 2002. L'art contemporain au Burkina Faso. Paris : L'Harmattan.

Kaboré, E. 1992/1993. La peinture contemporaine au Burkina Faso. Mémoire de maîtrise, Université de Ouagadougou, FLASHS, Département d'Histoire et Archéologie.

Kambou-Ferrand, J.M. 1993. Peuples voltaïques et conquête coloniale, 1885-1914, Burkina Faso. Paris : L'Harmattan.

Kiéthéga, J.B., 1993. La mise en place des peuples du Burkina Faso. Découverte du Burkina Faso, tome 1, Ouagadougou : Editions SEPIA, ADDB, 9-29.

Kouanda, A. 1986. L'historiographie du Burkina : un bilan. Connaissances du Burkina, CERLESHS : 4354.

Nikiéma, L., 2004/2005. La SNC, moyen d'affirmation de l'identité culturelle. Mémoire de maîtrise en Arts, Gestion et Administration Culturelle, Université de Ouagadougou, UFR/LAC, Filière AGAC.

Ouédraogo, D. 1989. Pour une réorganisation des Semaines Nationales de la Culture. Institut Culturel Africain, Centre Régional d'Action Culturelle, Section Conseillers Culturels, Dossier de Projet, Lomé.

Pibot, J. 2001. Les peintures murales des femmes kasséna du Burkina Faso. Paris : L’Harmattan

Sankara, T. 1983. Discours d'Orientation Politique (DOP). Prononcé le 2 octobre 1983 par le capitaine Thomas SANKARA, Président du CNR.

Traoré, D. 1985/1986. Le Musée National du Burkina : Approche historique. Mémoire de maîtrise d'histoire, Université de Ouagadougou, INSHUS.

Traoré-Sanogo, M. 1996. La Semaine Nationale de la Culture, Impacts socio-économiques (1983-1994). Mémoire de maîtrise d'histoire, Université de Ouagadougou, FLASHS.

UNESCO. 1981. L'affirmation de l'identité culturelle et la formation de la conscience nationale dans l'Afrique contemporaine. Paris : PUF.

Yaméogo, L. A.1989. Pour une administration culturelle déconcentrée au Burkina Faso. Institut Culturel Africain, Centre Régional d'Action Culturelle, Section Conseillers Culturels, Dossier de Projet, Lomé. 
Les arrêtés ministériels, les rapports, procès-verbaux et autres documents

Arrêté n²003395/MCAT/SG/SPSNC, portant Règlement Intérieur du Grand Prix National des Arts et des Lettres (GPNAL) de la 12eme édition de la SNC Bobo 2004. Catégorie A et B.

Arrêté n²005201/MCAT/SG/SPSNC, portant Règlement Intérieur du Grand Prix National des Arts et des Lettres (GPNAL) de la 13eme édition de la SNC "Bobo 2006". Catégorie B.

Arrêté n990011/MCC/SG/SPSNC, portant Règlement Intérieur du Grand Prix National des Arts et des Lettres (GPNAL) de la 10eme édition de la SNC "Bobo 2000".

Procès Verbal de délibération du jury de la catégorie B SNC Bobo 2004.

Rapport général du Comité technique de réflexion pour le Séminaire national sur le redimensionnement de la Semaine Nationale de la Culture du 16 au 17 septembre 2004.

Rapport général du séminaire sur la SNC du 5 au 10 avril 1993.

Rapport général du Séminaire sur le redimensionnement de la Semaine Nationale de la Culture du 21 au 22 octobre 2004

Rapport général sur la SNC Bobo 1994.

Rapport relatif à la Délibération du jury de la catégorie B du GPNAL de la Xe édition de la SNC Bobo 2000.

Résultats GPNAL de 1983 à 1996.

Statistiques sur la participation en arts plastiques de 1983 à 2004. 